\title{
LA INTERVENCIÓN COMO EJE TEÓRICO METODOLÓGICO DEL TRABAJO SOCIAL
}

\author{
Ángela María Quintero Velásquez'
}

Requerimos re-situar el Trabajo Social en una nueva época, dar cuenta de los procesos de conocimientos implícitos en la intervención profesional (Cifuentes, 2012).

\section{Resumen}

La intervención permea todos los procesos de Trabajo Social y le confiere una característica propia, que la distingue de otras carreras u ocupaciones. Es un concepto relacionado con las categorías de intervención, -indirecta y directa-, la metodología, el método, la técnica, la estrategia, la metodologías, la instrumentalidad, y los dispositivos instrumentales. Está presente desde el nacimiento de Trabajo Social como profesión, y pese a tener su origen en la medicina, ha evolucionado y ajustado al cambio de paradigma, para ser nombrado disciplinariamente como Intervención Social, como rasgo distintivo. Es la singularidad de la profesión, que la diferencia de otras modalidades de Intervención. Evoluciona al vaivén del giro paradigmático y de la consolidación disciplinaria. Coexisten intervenciones, tradicionales, dirigidas por modelos analíticos, funcionalistas, lineales e intervenciones modernas, guiadas por enunciados circulares, probabilísticos, integradores. En su esencia es un concepto resignificado y enaltecido en el ejercicio profesional, que integra propuestas democráticas para la construcción social, las dimensiones éticopolíticas y ético-técnicas y el compromiso con el desarrollo social en procesos sistemáticos y progresivos de diálogo de saberes, negociación cultural. Evoluciona con la época, y funge como eje

\footnotetext{
1 Trabajadora Social. Profesora Titular Jubilada Universidad de Antioquia, Medellín, Colombia.
} 
teórico metodológico del Trabajo Social, en convergencia con la investigación. Ilustrado esto con dos campos disciplinarios recientes, intervención familiar y socioterapia.

[Descriptores: intervención, Trabajo Social, intervención social, metodología, investigación, paradigma moderno del conocimiento].

\section{Abstract}

Intervention permeates all social work processes and gives it a characteristic that distinguishes it from other careers or occupations. It is a concept related to the categories of intervention - directly and indirectly- methodology, method, technique, strategy, methodologies, instrumentality, and instrumental devices. It is present from the birth of Social Work as a profession, and despite having its origin in medicine, it has evolved and adjusted to the paradigm shift, to be appointed as a Social Intervention discipline, as a feature. It is the uniqueness of the profession, that sets it apart from other forms of intervention. Evolves from theparadigm shift and disciplinary consolidation. Coexist interventions, traditional, directed by analytical models, functionalist, linear and modern interventions, guided by circular statements, probabilistic integrators. At its core is a redefined and lifted up at the practice concept that integrates democratic proposals for social construction, the ethical-political and ethicaltechnical dimensions and commitment to social development in systematic and progressive process knowledge dialogue, negotiation culture. Evolves with time, and serves as theoretical and methodological axis of Social Work, in convergence with the investigation. Illustrated this with two recent disciplinary fields, family intervention and social therapy.

[Keywords: speech, social work, social intervention, methodology, research, modern paradigm of knowledge].

\section{Introducción}

Uno de los propósitos del artículo es contribuir al fortalecimiento de los procesos de formación e identidad profesional. Se destaca el papel del Trabajador Social ${ }^{2}$, desde los orígenes de la disciplina y el tránsito conceptual y metodológico en la apropiación del

${ }^{2}$ El texto acoge la diversidad de denominaciones latinas: trabajador social, asistente social, promotor, tecnólogo, técnico, bachiller, licenciado, visitador social, servicio social que dan cuenta de la pluralidad continental. 
paradigma moderno del conocimiento, y en el carácter científico que acompaña este desarrollo centenario.

El análisis está focalizado en la intervención social, como la denominación propia, pero invocando la expansión del pensamiento hacia los escenarios multidisciplinarios, bajo la consideración de que el conocimiento no es exclusivo de ninguna profesión u oficio, sino que por su misma esencia, fundamenta la teoría y la praxis, en contextos universales y democráticos. Esta premisa es aplicada por excelencia en campos de actuación específicos, como familia, comunidad, políticas públicas, derechos humanos, movimientos sociales, entre otros, que comparten una base interdisciplinaria y su estudio, comprensión e intervención, sucede en el marco de la colaboración de las áreas del conocimiento, resaltando además los aportes técnicoconceptuales de Trabajo Social, tal como está reportado en la prolija bibliografía sobre dichos temas.

La intervención permea todos los procesos de Trabajo Social y le confiere una característica propia, que la distingue del conjunto de carreras u ocupaciones, que velan por el desarrollo humano. Un elemento sustancial es la relación dialéctica entre teoría y práctica, como partes de un todo, que en la modernidad no son dicotómicos, ni excluyentes, ni jerárquicos, ni contradictorios, sino que funcionan de manera convergente. De esta manera otras carreras universitarias han copiado los protocolos de prácticas e intervención de Trabajo Social, para no desaparecer, como unidades académicas ${ }^{3}$.

En esta lógica, el uso de los vocablos, debe estar argumentado, según el análisis documental y terminológico realizado en el contexto, y consignado en investigaciones lideradas por la profesión. Resaltando la contribución del Trabajo Social latino a otras áreas, todo lo cual neutraliza el uso ideológico, empírico y subjetivo de los conceptos.

Asimismo, destaca la importancia de la investigación como un reto para la formación integral en las ciencias sociales, enfatizando los aportes de Trabajo Social con las metodologías cualitativas y hermenéuticas. Vr.gr: sistematización, diccionarios y tesauros especializados, investigación acción participativa, estados del arte, glosarios, entre otros. Cuyos productos

\footnotetext{
${ }^{3}$ Ver la propuesta de intervención sociológica de Alain Tourine, que se adapta en la región.
} 
contribuyen a generar conocimiento científico, específico y multidisciplinario.

\section{Marcas de contexto sobre intervención.}

El vocablo de intervención, ha sido difamado, subutilizado, menospreciado, y contra todos los pronósticos, en gran parte por sectores de Trabajo Social. No obstante, la identidad e historia profesional, recoge esta denominación y en las postrimerías del siglo $\mathrm{XX}$, hace una revisión crítica de la evolucion y aplicación del término. Habida cuenta de su origen en la medicina y psicología, el Seminario Latinoamericano en Santiago de Chile, 1998, enaltece la acepción de Intervención Social:

...el concepto fue resignificado en la medida en que crecían las preocupaciones generadas por los efectos del amplio alcance de la nueva organización industrial y urbana que modifico las condiciones de vida durante los siglos XIX y XX. Estos nuevos problemas y demandas son los que en general identificamos colectivamente como la "cuestion social (Eroles, 2005, pp.107-8).

Es la singularidad de la profesión, que la diferencia de otras modalidades de intervención, -sociológica, piscológica, terapéutica, médica, jurídica, económica, antropológica, pedagógica, entre otras-. Evoluciona al vaivén del giro paradigmático y de la consolidación disciplinaria.Varias voces contribuyen al debate continental, con premisas científicas y de validación terminológica. Por ejemplo, según Quintero Velásquez (2004):

En el siglo de la incertidumbre, emerge una discusión que compete a todas las ciencias sociales y humanas: el replanteamiento del concepto de intervención, término procedente de la medicina, de carácter directivo, intrusivo, por el cual se conciben denominaciones más acordes con los procesos colaborativos y cocreadores, pero que a su vez, recojan la tradición de Trabajo Social (pp. 29 - 31).

La discusión reciente sobre el concepto de intervención permea a Trabajo Social. El panorama latino refleja diversas tendencias: Clásicas de corte funcionalista/positivista, como la expuesta por Myriam Valjalo (según citado por Quintero Velásquez, 2004):

El trabajo social es una intervención en el sentido que "intervenir" significa tomar parte de una acción con la intención de influenciarla. La intervención del trabajador social consiste en permitir a la persona/sujeto de desarrollar sus capacidades, de ayudarla a modificar 
su situación y finalmente a ayudarlo a resolver sus problemas.

Otras a tono con la tradición de la profesión en la acción social, sustentada por Luz Marina Pava y colaboradores (2001):

Trabajo Social en su Intervención es una forma de acción social, consciente y deliberada, realizada de manera expresa; integra los procedimientos operativos en un marco referencial teórico y/o empírico y en los supuestos ideológicos, políticos, filosóficos que las sustentan. La intervención supone un proceso, a partir del conocimiento que desde la misma problemática se posee: se identifica, busca una posición de equilibrio para evitar desviaciones especulativas o riesgos innecesarios; reconoce diferentes realidades subjetivas construidas mediante las representaciones y la comprensión interna de los hechos, desde perspectivas particularizantes y de teorías sociales que juegan un papel explicativo y guían el conocimiento, el proceso y los resultados. Algunos se refieren a la intervención profesional como ejercicio profesional, que expresa un saber hacer especializado.

Olga Lucía Vélez (según citado en Quintero Velásquez, 2004), declara:

En sintonía con lo anterior y buscando contribuir con la apertura conceptual que tiene que operarse en el lenguaje profesional contemporáneo (teórico y metodológico), propongo la eliminación del término "intervención", reemplazándolo por el de actuación. Entendiendo la misma como el conjunto de actos, prácticas y procesos condicionados por interacciones y mediaciones sociales (internas y externas) que estructuran la especificidad de Trabajo Social, y cuya elección no es ajena a las nociones, visiones o posturas que sobre la realidad, la profesión y la acción social se tengan. La actuación profesional tiene un marco intencional estructural y contextual que la hacen posible. (p.54) 
Desde hace algunos años, la Sistémica en el campo específico de familia, sugiere interacción, término invocado en programas institucionales del ICBF${ }^{4}$ :

...he ido descubriendo que las palabras dicen cosas y es que trabajar con niños y niñas desvinculados del conflicto armado desde una perspectiva de intervención, genera problemas muy serios, pensar, soñar y sentir el Sistema Nacional de Bienestar Familiar, no ha funcionado porque se ha mirado desde una perspectiva de intervención, entonces como el seminario se llama Intervención con niñez y juventud en situación de especial vulnerabilidad, yo quisiera proponer que al final del seminario..., mirar la posibilidad de dejar de hablar de intervenciones y empezar a hablar de interacciones" [subrayado personal] (Urrutia, según citado en Quintero Velásquez, 2004).

Por otro lado, Barg, (según citado en Quintero Velásquez, 2004) señala.

Estamos planteando la necesidad de usar la investigación como herramienta cotidiana en la intervención, dejando de lado actos voluntaristas para introducir aquellos que constituyen situaciones de estudio, de investigación, de indagación, conociendo 'el tiempo-ahora' de la familia, su historia, sus tradiciones, su situación actual y anticipar la futura. (p. 105)

Según lo argumentado, coexisten intervenciones tradicionales, dirigidas por modelos analíticos, funcionalistas, lineales e intervenciones modernas, guiadas por enunciados circulares, probabilísticos, integradores. ¿Cómo operar? Depende de la formación profesional, de la visión personal de mundo, de la misión institucional, del contexto cultural, por lo cual no es conveniente excluir el uno y el otro, sino entenderlos como propuestas ancladas, las unas en el modelo clásico, analítico del conocimiento (eficaz en asuntos simples) y las otras, en el paradigma sintético, sistémico (útil en eventos complejos como lo es la sociedad actual). Sin ánimo de polemizar, ni de profundizar al respecto, pues correspondería a otro razonamiento, se reseñan algunas de las denominaciones propuestas para suplantar el concepto de intervención con familias, con la noción de: sistema del lenguaje colaborativo,

${ }^{4}$ Instituto Colombiano de Bienestar Familiar: ente rector de instituciones del Sistema Nacional de Bienestar Familiar de Colombia. En México, Sistema Nacional para el Desarrollo Integral de la Familia (SNDIF). 
entrevista etnográfica, estrategia colaborativa, conversación terapéutica, consulta sistémica, estrategia de aproximación.

Las ideas expuestas sobre intervención, proponen asumir los avances y la construcción disciplinaria en Trabajo Social bajo los preceptos modernos del enfoque ecosistémico, en escenarios de convergencia y de cocreación. Acordes con el mandato gremial de ejercer la profesión analizando, contrastando, develando y denunciando los elevados e injustos índices de exclusión, pobreza, vulneración de los derechos e inequidad, que caracterizan el continente.

La acepción moderna de intervención social, entroniza con la tradición metodológica de Trabajo Social, en tanto plantea las categorías de intervención, -indirecta y directa-, metodología y método de intervención. Reforzando elementos consustanciales, a saber: técnicas, estrategias, metodologías, instrumentalidad, dispositivos instrumentales. De acuerdo con Cifuentes (2012), "en la intervención se relacionan dialécticamente conocimiento, acción, transformación; se desencadenan dinámicas de participación y empoderamiento en procesos regionales y locales" (p. 5).

Resultado de investigaciones documentales y terminológicas, y foros gremiales y universitarios, los conceptos adquieren una dimensión sincrónica, que da cuenta de las tendencias contemporáneas y del análisis del contexto. La propuesta inherente en este ejercicio, es de contribuir a la expansión del pensamiento en ámbitos latinos, que valoricen la producción intelectual de Trabajo Social y de manera colaborativa, recree las nuevas tendencias en intervención, como eje rector de la profesión.

Las referencias y argumentación que sustentan la reflexión, fluctúan entre autores latinoamericanos destacados como Carballeda, Cifuentes, De Robertis, Quintero, Universidad de Antioquia ${ }^{5}$ (Medellín-Colombia), Ander-Egg, Eroles, Molina, entre otros. A manera de ilustración, se presentan dos definiciones básicas acogidas por la comunidad académica en Colombia, consignadas en libros y ponencias de circulación internacional.

${ }^{5}$ Grupo de Investigación, Intervención Social, GIIS, en la línea de investigación Trabajo Social e intervención profesional. Destacando los aportes de la líder originaria del grupo, profesora Mgs Paula Andrea Vargas y un prolífero repertorio de artículos, ponencias, libros e investigaciones del tema, en la última década 
Intervención. (Intervention, Intervention)

Proceso sistemático y coherente que se orienta fundamentalmente a la modificación/transformación de alguna problemática social que puede ser de orden individual, institucional o colectiva. Es una acción específica del trabajador(a) social con relación a los sistemas o procesos humanos para producir cambios que desarrollen la capacidad de reacción e iniciativa del usuario, para estimularlo a recuperar su propia capacidad de llevar a cabo acciones, de emprender actividades que sirvan para eliminar las causas de su situación de malestar. La intervención se relaciona con un querer actuar. Es el 'qué hacer', pero también el 'cómo hacerlo' de la mejor forma posible. Las formas de intervención pueden ser numerosas y variadas, mas lo esencial no es solamente definirlas, sino determinar a partir de qué elementos se efectúa tal o cual intervención teniendo en cuenta que los elementos analíticos que constituyen esta, tienen que ver con cambios existentes en el contexto, las diversas perspectivas de teorías sociales, los enfoques epistemológicos, y los marcos éticamente valorados por las sociedades. No puede haber intervención sin un planteamiento teórico o conceptual previo que tome en cuenta las consecuencias políticas y éticas de tales actos (Cardona, Montoya, \& Zapata, 2002, pp. 78-79).

Intervención social. (Social intervention, Intervention sociale) Proceso sistemático y coherente que se lleva a cabo frente a problemáticas sociales no resueltas, que se desarrolla a través de acciones con carácter de educar y generar procesos organizativos, que llevan implícitos una ideología orientada fundamentalmente a la modificación y transformación de las maneras de ver, actuar y sentir de los individuos en su inserción social. Existen modalidades de intervención, ya sea desde arriba, por medio de organismos de planificación y del orden institucional, o desde abajo, propiciada por las organizaciones comunitarias de base. (Cardona, Montoya, \& Zapata, 2002, pp. 78-79).

Definición ampliada por Ander-Egg, en la pragmática de su pensamiento:

Intervención social. Esta expresión se ha introducido en el campo de las prácticas sociales para designar el conjunto de actividades realizadas de forma más o menos sistemática y organizada para actuar sobre un aspecto de la realidad social con el propósito de producir un impacto determinando. Toda forma de intervención social se apoya en referentes técnicos, necesita de métodos y 
técnicas de acción, y tiene una intencionalidad que viene dada por el marco ideológico, político o filosófico/operativo, se configura de acuerdo con un modelo cuatridimensional que comporta el sujeto de intervención, aquello sobre lo que se interviene y la categoría o forma de intervención (Ander-Egg, 2009, pp. 63-64).

De una u otra manera, gran parte de las definiciones, artículos, textos e investigaciones sobre el tema, están sincronizados con el método, la metodología, la estrategia y la técnica. No son categorías abstractas, dicotómicas, aisladas, sino que hacen parte del fuero disciplinario y del acervo tradicional, que ha distinguido a la profesión desde sus orígenes, en su evolución acorde con el cambio de época.

En correspondencia, la formación en pregrado (profesionista en México), y posgrado, exige el abordaje científico de estos dominios disciplinarios, en su carácter sistémico, circular, integral, diacrónico y sincrónico. El paradigma moderno del conocimiento ofrece diversas opciones teóricas, compatibles con la misión y los propósitos universales de Trabajo Social, pero lo fundamental desde la investigación y la epistemología, es la convergencia entre las Ciencias Sociales clásicas y los enfoques y teorías contemporáneos, que articulados con la flexibilidad disciplinaria, recrean el sustrato metodológico y la potencian en constructos colaborativos.

En el 2008, el Consejo Nacional de Educación en Trabajo Social (CONETS) indica que a pesar de su tradición histórica, el concepto de intervención es polémico: ...Conviene resignificar que se utiliza en diversas profesiones y disciplinas aplicadas. Las perspectivas constructiva, sistémica y compleja, aportan a replantearla críticamente, posicionarnos estratégicamente; desde concepciones acordes con desarrollos teóricos y propuestas democráticas para la construcción social. Cifuentes (2012) implica "el integrar las dimensiones ético-políticas y ético-técnicas, al compromiso con el desarrollo social en procesos sistemáticos y progresivos de diálogo de saberes, negociación cultural" ( $p$. 30 La metodología es el modo como se elabora o desarrolla un proceso de intervención. Entraña una posición científica, una visión teórica y una opción ideológica y a través de ella, el operador social comprende el contexto y propicia alternativas para estudiar y abordar los fenómenos humanos, en su esencia ontológica, filogenética y epigenética. La conforman los métodos de la disciplina y está relacionada con la percepción de los 
sujetos sociales y la organización de los postulados teóricos que sustentan el método. De esta manera, incluye el método, los sujetos sociales y al marco teórico como soporte de la práctica.

Metodología en Trabajo Social. (Social work methodology, Méthodologie en travail social)

Proceso holístico y complejo que se inicia analíticamente en el instante en que los lineamientos se configuran como elementos lógicamente interrelacionados, a través de la aplicación de técnicas enfocadas filosófica e ideológicamente, que permiten coadyuvar a los procesos de cambio, para sustentar acciones enfocadas a determinados objetivos, confirmándolos o negándolos (Cardona, Montoya, \& Zapata, 2002, p. 91).

La característica de la época, en su complejidad, incertidumbre, dialéctica, diversidad, exige que el profesional de Trabajo Social, innove, recree, pedagogías ciudadanas y políticas, estrategias y dispositivos técnico-instrumentales, acordes con la evolución del planeta y sus habitantes. Esto es un cambio de paradigma desde lo individual y lo colectivo, para impactar positivamente a la sociedad y generar dominios que garanticen y coadyuven en la calidad de vida y el respeto de los derechos mínimos, más allá del discurso retórico. Es un compromiso profesional, académico, gremial y ciudadano, en el devenir humano.

\section{Retos de la intervención}

Las preguntas sobre el origen del conocimiento científico en las ciencias humanas y sociales, las proposiciones teóricas que están insertas en los modelos de intervención, los fundamentos epistémicos y los problemas éticos metodológicos en el Trabajo Social, nos invitan a pensar en la posibilidad de una epistemología del Trabajo Social. Según Reyes (2012) "esto ayudará construir una propuesta que permita vislumbrar nuevas cartografías epistemológicas para potenciar la intervención y los fundamentos teóricos que sustentan la práctica del trabajador social (pp. 1-3).

El necesario debate y replanteamiento del concepto de intervención, en cualquiera de sus dimensiones, está ligado inexorablemente a los dispositivos metodológicos, estratégicos y técnico-instrumentales. La intervención de Trabajo Social, está situada fundamentalmente en los contextos clínicos (terapia familiar, cambios de segundo orden) y contextos no clínicos 
(orientación, recepción, evaluación, prevención, promoción, educación, cambios de primer orden).

Honrando la tradición de Trabajo Social y su esencia primigenia, no se trata de desconocer, excluir, ridiculizar, su vasta experiencia y aportes en la asistencia y ayuda humanitaria y en proyectos de beneficencia pública, sino en encauzar este capital metodológico para entronizarlo en la época con las innovaciones propias de los tiempos y con los recursos uni y multidisciplinarios que permitan la atención integral de los complejos fenómenos humanos en programas preventivos, promocionales y educativos. Esto, bajo los enfoques de derecho, de género, poblacional, diferencial, territorial y multicultural.

La intervención social debe tener como propósito la transformación de la realidad a partir de su lectura crítica y de prácticas orientadas a la formación de sujetos sociales, políticos y éticos capaces de generar procesos sociales de concertación, diálogo y reivindicación en torno a los derechos humanos. Nos dicen Díaz, Pérez, y Páez (2013):

...las intervenciones sociales cobran vital importancia hacia el impulso de procesos sociales, formativos, de promoción y acompañamiento, que potencien ciudadanos autogestores, participantes y autónomos y corresponsables con el desarrollo y la justicia social en su contexto local. (p. 21)

En los tiempos ultra/posmodernos de la incertidumbre, del caos, de la ambigüedad, del desorden, como categorías científicas, la propuesta central es transformar la visión del mundo, desde un paradigma analítico, reduccionista, lineal, determinista, por una cosmovisión holística, probabilista, circular, integradora. Esto implica trascender los conocimientos e información digital, y pensar de otra manera para intervenir en correspondencia.

Los dominios científicos, fundamentados por el Trabajo Social en América Latina, asumen el enfoque de convergencia, avanzan hacia el lenguaje especializado, la circularidad de los procesos, los profesionales polivalentes, innovadores, creativos, resilientes, comprometidos ética y políticamente. La perspectiva ético-política es entendida, como coherencia entre el proyecto vital y profesional, la alteridad y el respeto por el otro, la diversidad en múltiples espacios (familia, sexualidad, territorial, étnica). Implica neutralizar y superar el culto a la personalidad, al fundamentalismo, a los guetos académicos y gremiales, que excluyen a quienes postulan posiciones diferentes. 
En la Americanidad que propone Carballeda, -Argentina $-{ }^{6}$, esto es rebasar la endogamia disciplinaria y geográfica. Ello conlleva la expansión del pensamiento y del conocimiento, el deslinde de fronteras,- conocer del Sur al Norte del continente, de manera bidireccional-, y trascender el dogmatismo ideológico, proselitista y empírico. Al igual que su bagaje conceptual, el Trabajo Social Contemporáneo, expone categorías y constructos, que rescatan su tradición, con los escenarios de intervención que requieren los tiempos. Con ello ratificamos el precepto de que la Intervención Social, evoluciona con la época, y funge como eje teórico metodológico del Trabajo Social. En las relaciones y los entornos de una época de cambio, en la construcción disciplinaria y la consolidación de la identidad, siempre es recomendable e importante, analizar la acción profesional, en una perspectiva multidisciplinaria, rescatando la producción intelectual en lengua española.

El proceso de intervención del trabajador o trabajadora social puede representarse en forma de una espiral. Consideramos que este gráfico da cuenta a la vez de la complejidad y del movimiento, de los aspectos específicos de cada etapa del método y de su simultaneidad, así como del desarrollo de la acción en el tiempo (De Robertis \& Pascal, 2007). Para la ilustración, se enuncian algunos campos de actuación disciplinaria con crecimiento exponencial en las últimas décadas, debido a la fundamentación científica e investigación, que provee de conocimientos y un repertorio convalidado y aplicado en temas tan disímiles, pero universales, como:

- Enlaces socio-jurídicos: papel protagónico del Trabajo Social en las reformas constitucionales, legales y estatales. Enfatizando los Niños, Niñas y AdolescentesNNAJ. Matrimonios igualitarios, adultos mayores.

- Trabajo Social forense. Peritaje social. Dictamen pericial.

- Trabajo Social familiar. Diversidad familiar. Violencias de toda índole.

- Movilidad social: migraciones intracitadinas, nacionales,

6 En: V Encuentro GIITS- del Grupo Interuniversitario de Investigadores en Trabajo Social, Santiago de Chile, 2013 (Notas personales). 
transnacionales. Desplazamiento forzado: personas, familias y comunidades en situación de desplazamiento. Familias en situación de transnacionalidad.

- Gerencia social y del desarrollo. Responsabilidad social. Precarización e informalidad Laboral.

- Derechos humanos: todo el espectro de los derechos de cuarta generación. Verificación, garantía y restablecimiento. Sujetos prevalentes de derechos. Políticas públicas: participación en el diseño, ejecución, y evaluación de programas y proyectos de orden ciudadano.

- Personas en situación de vulnerabilidad, riesgo social, exclusión y discapacidad.

- Movimientos sociales. Participación comunitaria y política.

- Enfoque de género: Género en el lenguaje. Feminización de la pobreza y la migración.

A modo de ejemplo final, presentamos dos definiciones que sustentan, el enlace entre investigación e intervención:

Intervención familiar (Family intervention)

Proceso de interacción entre el profesional y la familia para el abordaje de una situación familiar que requiere una acción experta, la cual puede ser de asesoría, sostén, control, tutela, mediación o terapia; por medio de la creación conjunta de contextos que expandan las acciones, las cogniciones, los territorios afectivos y nuevas condiciones relacionales, dentro de las cuales las familias generen sus propios recursos y así invertir la dirección disfuncional por la que atraviesan. Se requiere establecer la naturaleza de la situación y la necesidad de profundizar el tratamiento, $\mathrm{o}$ atender la disfunción en algunas sesiones de orientación. Las intervenciones son conducidas por los servicios sociales, educativos, sanitarios, religiosos, comunitarios, psicológicos, jurídicos, organismos gubernamentales y no gubernamentales. Operan en los contextos no clínicos y en los contextos clínicos (Quintero Velásquez, 2007).

El término socioterapia, es el modelo de intervención que tiene como meta la mejora del mantenimiento del equilibrio sociorelacional de los sistemas humanos, llámese familia, grupo 
o comunidad y cuya práctica es la aplicación científica de la teoría y los métodos de tratamiento del Trabajo Social, interviniendo específicamente en los espacios intervinculares, activando los recursos sanos pre-existentes y gestando los no desarrollados que, conducirán a la reducción de las causas que sostienen la problemática. Desde esta perspectiva, podemos pensar en el Trabajo Social terapéutico con una mirada renovada. Un adecuado 'soplo de aire fresco' que da constancia del crecimiento y evolución del Trabajo Social, como disciplina que brinda 'razón de sí' (Chadi, 2013).

Esto es situar la intervención como eje teórico metodológico del Trabajo Social, en convergencia con la investigación. El fenómeno de la producción de conocimiento está asociado con la construcción de comunidad académica en torno a los debates contemporáneos y a los enfoques de convergencia, que privilegien la concertación, el objeto de intervención y la identidad, sin menoscabo de la multidisciplinariedad que es el norte de toda la acción social y de la simetria científica. Sistematizar la intervención profesional de Trabajo Social aporta a la producción intencionada de conocimientos sobre y desde la práctica, su reconstrucción pertinente, de modo que podamos contextualizarla histórica y socialmente e interpretarla, al asumir el reto profesional de la autorreflexión sobre la intervención profesional (Cifuentes, 2012). La investigación básica, fundamenta la intervención: la producción científica permite superar empirismo y posiciones, ideológicas, empírica -analíticas, excluyentes, huecas, vacías de argumentación y epistemología. En correspondencia con las divergencias ideológicas, políticas y disciplinarias, el debate actual replantea la definición de Trabajo Social y por ende, del término de intervención. Pero sin ánimo de ser concluyentes, ni de polemizar, es significativo el pronunciamiento de la Dirección Ejecutiva de ALAEITS (2013), al respecto:

Impulsamos un Trabajo Social que se profesionalice y defienda su campo de intervención cada vez más y con mayores conocimientos, habilidades y destrezas, pero lo hacemos desde la concepción de un Trabajo Social comprometido con la resolución de los problemas y situaciones de su época. Invitamos a reconocer al Trabajo Social como profesión y como área de conocimiento, ya que esta última nos liga con la dimensión intelectual de la profesión expresada en su capacidad de investigar la realidad y analizar los fenómenos como parte de 
la dinámica de lo real, produciendo un pensamiento crítico; queremos trascender la condición de Trabajo Social como una práctica profesional destinada solo a intervenir y sistematizar, su hacer, por ello reivindicamos e impulsamos la producción de conocimiento como dimensión constitutiva de la profesión, consideramos que ello nos permitirá ejercitar una relativa autonomía teórica, práctica y política (pp. 5-6). 


\section{REFERENCIAS}

Ander-Egg, Ezequiel. (2009). Léxico del Trabajo Social y los Servicios Sociales. Asunción: Centro Gráfico.

ALAEITS-Asociación Latinoamericana de Enseñanza e Investigación en Trabajo Social. Dirección Ejecutiva 2012-2013. (Octubre 2013). Algunas consideraciones y aportes en torno a la Definición Internacional de Trabajo Social. Córdoba, Argentina: Autor

Cardona, Bertha Nelly, Montoya, Gloria, \& Zapata, Cecilia Inés. (2002). Diccionario Especializado de Trabajo Social. Medellín: Universidad de Antioquia, Centro de Investigaciones Sociales y Humanas-CIHS, Digital.

Cifuentes Gil, Rosa María. (2012). Trabajo social: integración metodológica, sistematización e interdisciplinariedad. En: IV Congreso Internacional de Trabajo Social: Trabajo Social y transdisciplinariedad en el siglo XXI. México: Universidad Autónoma de Juárez, Programa de Licenciatura en Trabajo Social

Chadi, Mónica. (2013). ElTrabajo Socialy Sus Alcances Socioterapéuticos. En: Aportes, Año 4, No 33. Video-Conferencia V Congreso Internacional de Trabajo Social. Maracaibo, Venezuela: Universidad Zulia- Escuela de Trabajo Social.

De Robertis, Cristina, \& Pascal, Henri. (2007). La intervención colectiva en Trabajo Social. La acción con grupos y comunidades. Buenos Aires: Lumen-Hvmanitas.

Díaz Flórez, Yudis del Carmen, Pérez Mendoza, Liliana, \& Páez Rodríguez, Graciela Isabel. (2013). Intervención social: Representaciones sociales y prácticas de estudiantes de Trabajo Social. Revista Análisis, XIV, (1), 20-40.

Gómez Gómez, Francisco. (2012). “La intervención sistémica. Un nuevo paradigma filosófico y pedagógico". En Ballester Brage, LL. \& Colom Cañellas, A.: Intervención sistémica en familias y en organizaciones socioeducativas. Barcelona: Octaedro.

Eroles, Carlos. (2005). Glosario de los temas fundamentales en Trabajo Social. Buenos Aires: Artes Gráficas.

Quintero Velásquez, Ángela María. (2004). El Trabajo Social Familiar y el Enfoque Sistémico. Buenos Aires: Lumen/Hvmanitas.

Quintero Velásquez, Ángela María. (2007).Diccionario Especializado en Familia y Género. Buenos Aires: Lumen- Hvmanitas.

Quintero Velásquez, Ángela María. (2012). Reflexiones sobre la intervención socio-familiar. En: Aportes 32. Buenos Aires. www. monicachadi.com.ar

Reyes Barría, Diego. Epistemología del Trabajo Social: Definiciones, Complejidad e Identidad. Boletín Electrónico Surá \# 190, Octubre 2012. Escuela de Trabajo Social, Universidad de Costa Rica. www.ts.ucr.ac.cr

UNAM-Universidad Nacional Autónoma de México. Unidad de Posgrado. Encuentro Intervención del Trabajo Social Contemporáneo: una mirada internacional. México. Distrito Federal. Noviembre 2013. 